\title{
Effects of visual stimulus degradation, S-R compatibility, and foreperiod duration on choice reaction time and movement time
}

\author{
H. W. FROWEIN and A. F. SANDERS \\ Institute for Perception TNO, Kampweg 5, Soesterberg, The Netherlands
}

\begin{abstract}
In a 2 by 2 by 2 factorial experiment, 12 subjects carried out a choice reaction time task. Independent variables were foreperiod duration (1.5 sec vs. $10.5 \mathrm{sec})$, stimulus degradation, and stimulus-response (S-R) compatibility. The speed of the response was measured in terms of reaction time (RT) and movement time (MT). The data showed additive effects of foreperiod duration (FPD), S-R compatibility, and stimulus degradation on RT. None of these variables had an effect on the MT. This is consistent with the hypothesis that stimulus encoding, response selection, and response execution represent independent processing stages, and suggests that FPD affects none of these stages.
\end{abstract}

When a subject has to make a rapid response to a signal, reaction time (RT) is shortened if the reaction signal is preceded by a warning signal. As the foreperiod duration (FPD) between warning signal and reaction signal decreases, the RT decreases until some optimal foreperiod is reached (e.g., Alegria, 1974; Bertelson, 1967; Sanders, 1972). Although several explanations have been proposed (e.g., Posner, Klein, Summers, \& Buggie, 1973; Sanders, 1977), the locus of the effect in the information flow has not as yet been conclusively determined.

A systematic approach to investigate this issue could be through the additive factors method (Sternberg, 1969), which assumes that different task variables affect different processing stages if they show additive contributions to RT, while an interaction between the effects of different task variables is assumed to indicate that these variables affect the same processing stage. Although the assumption that additivity implies separate processing stages has recently been criticized (Taylor, 1976), application of the additive factor method to the existing data shows a fairly consistent picture of processing stages.

Thus, regarding FPD, additive contributions have been observed with visual stimulus intensity (Raab, Fehrer, \& Hershenson, 1961; Sanders, 1975) and with stimulus-response (S-R) compatibility (Posner et al., 1973; Sanders, 1977). Considering this in conjunction with the finding that both stimulus intensity and stimulus degradation show additive effects with S-R compatibility (Sanders, 1977; Sternberg, 1969), it would

The investigation was supported by the I:oundation for Medical Research (FUNGO), which is subsidized by the Netherlands Organization of Pure Research (ZWO). The authors wish to thank A. J. Krul for carrying out the statistical computations and J. Th. Eernst for the construction of the apparatus. seem that response selection constitutes a processing stage that is independent of stimulus processing and that FPD affects neither encoding nor response selection. The present experiment constitutes a further test of this conclusion by investigating the effects of FPD, stimulus degradation, and S-R compatibility in the same reaction task.

Second, the experiment investigated whether FPD and the other two task variables have an effect on response execution. Fitts (1954) observed that movement time (MT) was not affected by the number of alternatives in a choice reaction task, suggesting that RT and MT represent independent processes and that MT can be used as a measure of response execution independent of information processing. For this reason, both RT and MT were measured in the present experiment. Thus, if information processing and response execution constitute independent serial stages, there should be no effect of stimulus degradation and S-R compatibility on the MT. Furthermore, an effect of FPD on the MT could then be interpreted as an effect of FPD on response execution as distinct from information processing.

\section{METHOD}

\section{Subjects}

The subjects were 12 male students from the University of Utrecht, with an age range from 20 to 30 years. The subjects were paid Hfl. 60 for participating in the experiment.

\section{Task and Apparatus}

The task was a visual four-choice reaction task with RT and MT as the response measures. The subject was seated in a soundattenuating cubicle at a sloping desk. The stimulus situation is schematically presented in Figure 1. The visual signals consisted of flashes generated by a Nixie tube situated about $1 \mathrm{~m}$ in front of the subject. The warning signal $\left(\mathrm{S}_{1}\right)$ consisted of a $500-\mathrm{msec}$ flash of the Nixie tube with all elements activated, and the imperative signal $\left(S_{2}\right)$ consisted of a 200 -msec flash of a diagonal 


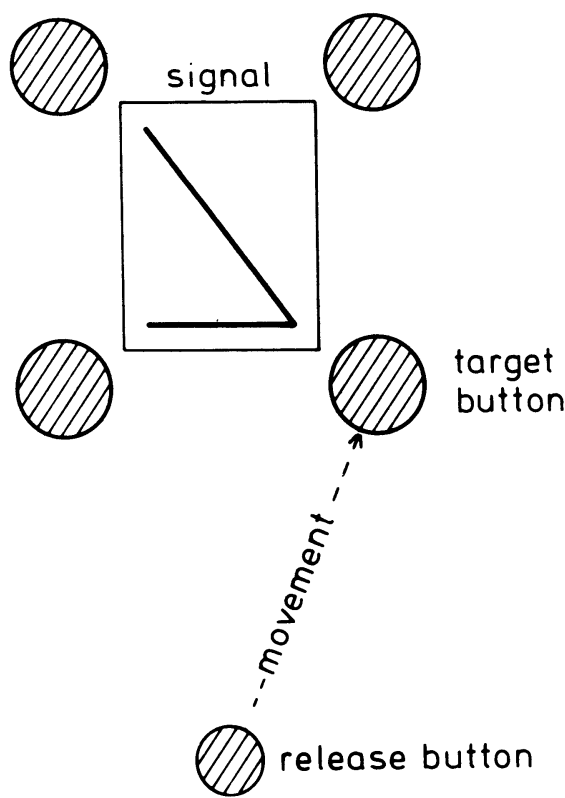

Figure 1. Schematic representation of the stimulus situation.

and horizontal line meeting at one of the four corners of the Nixie tube. The FPD, defined as the interval between the onset of $S_{1}$ and the onset of $S_{2}$, was either 1.5 or $10.5 \mathrm{sec}$. The intertrial interval was always $5 \mathrm{sec}$. The index finger of the subject's preferred hand was resting on the release button, and his task was to make a movement with his index finger to press one of the four target buttons. The distance between the release button and the target button was $13 \mathrm{~cm}$ for the two bottom target buttons and $20 \mathrm{~cm}$ for the two top target buttons. The subjects were specifically instructed that the warning signal served to prepare for a fast response and that, once the movement was initiated, it should be made as rapidly as possible without hesitation about which button to press. They were also instructed to work as accurately as possible.

Compatibility was varied in the following manner. The correct target button in the compatible condition was the joining point of the two lines (the bottom right button in Figure 1), while the correct response in the incompatible condition was to press the next target button going in counterclockwise direction (in the illustration shown in Figure 1, the upper right button). Stimulus degradation was achieved by superimposing a photonegative with a visual noise pattern upon the surface of the Nixie tube. The noise pattern consisted of a cluster of black nonsense shapes, each averaging about $1 \mathrm{~mm}$ in diam. The light-to-dark ratio was about $30 \%$. To avoid differences in light intensity between the two conditions, a similar photonegative without visual noise was used for the undegraded condition. The preprogrammed signal presentation and the registration of the responses was performed by the PSARP system (Van Doorne \& Sanders, 1968). This allowed the measurement of RT, defined as the interval between the onset of the imperative signal and the release of the release button, and MT which was defined as the interval between the release of the release button and the pressing of the target button.

\section{Design and Procedure}

A 2 by 2 by 2 factorial design was used, with stimulus degradation (undegraded vs. degraded), S-R compatibility (compatible vs. incompatible), and FPD (1.5 sec vs. $10.5 \mathrm{sec})$ as the independent variables. Experimental conditions were varied between blocks of 20 trials, of which the first 5 were regarded as warm-up trials not to be used in the data analysis. The order of presentation of the compatibility and degradation conditions was counterbalanced in the manner of a Latin square design, with degradation nested within compatibility; the order of presentation of the two FPDs was partially counterbalanced within degradation conditions. Two blocks with different foreperiods, but the same compatibility and degradation conditions, were carried out one after the other with a 2-min rest period in between, while blocks differing in compatibility and/or degradation were always separated by a $20-\mathrm{min}$ rest period. Subjects were trained for 1 whole day prior to the experiment. On the day of the experiment the whole sequence of conditions was run twice so that there were two blocks for each experimental condition. Hence, the total number of trials used in the analysis was: 2 (FPD) by 2 (compatibility) by 2 (degradation) by 12 (subjects) by 2 (blocks) by 15 (trials per block) $=2,880$ trials.

\section{RESULTS}

For each experimental condition, the data of the two blocks were combined, and the average RTs and MTs were pooled over different response keys and computed for each subject. The group means are shown in Figure 2.

The analysis of variance for RTs showed significant effects of stimulus degradation $[F(1,11)=56.7$, $\mathrm{p}<.01]$, S-R compatibility $[\mathrm{F}(1,11)=380, \mathrm{p}<.01]$, and FPD $[F(1,11)=28.1, p<.01]$. There were no significant interaction effects on RT. A separate analysis of variance on the MTs showed no significant effects of degradation or FPD. Although Figure 2 suggests a small

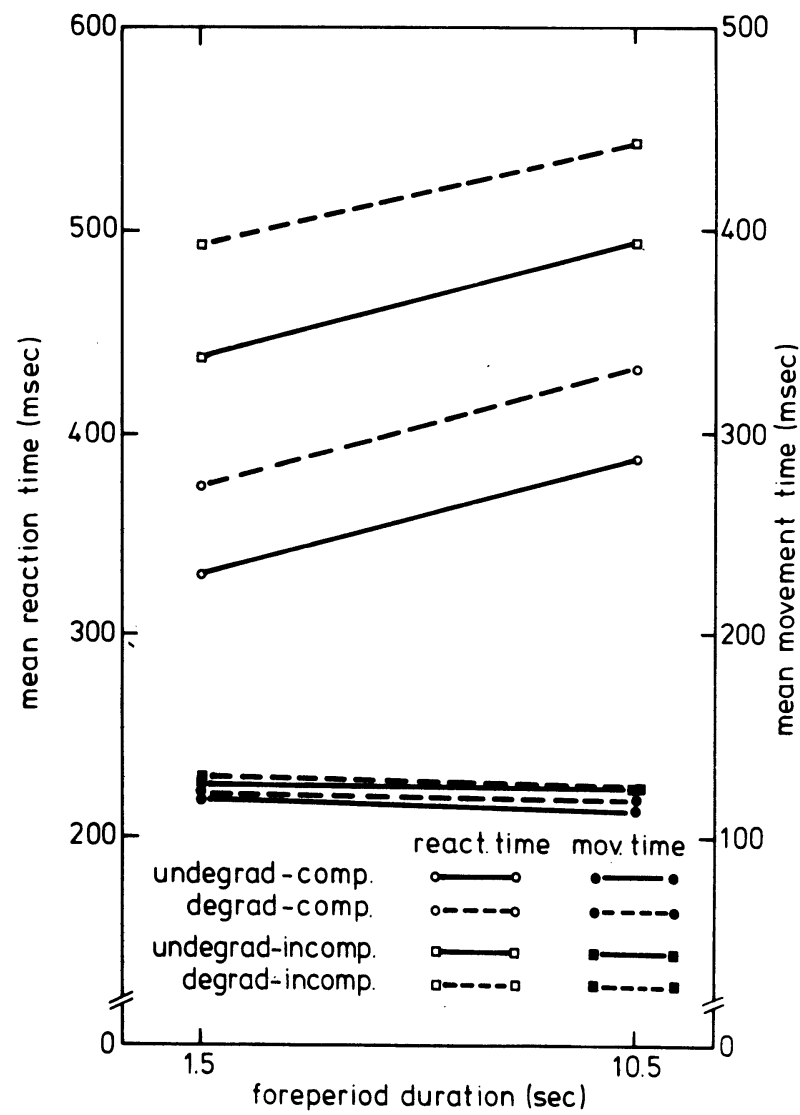

Figure 2. Reaction time and movement time as a function of S-R compatibility, stimulus degradation, and foreperiod duration. 
Table 1

Mean Percentages of Errors

\begin{tabular}{lcc}
\hline \multicolumn{1}{c}{ Foreperiod } & $1.5 \mathrm{sec}$ & $10.5 \mathrm{sec}$ \\
\hline Compatible-Undegraded & 3.9 & 4.2 \\
Compatible-Degraded & 5.6 & 6.9 \\
Incompatible-Undegraded & 6.9 & 7.5 \\
Incompatible-Degraded & 7.5 & 8.9 \\
\hline
\end{tabular}

effect of S-R compatibility on the MT, this effect was not statistically significant $[F(1,11)=3.45]$. Interactions were also nonsignificant.

The error scores presented in Table 1 also include trials in which the subject failed to press the target button and trials in which the subject was assumed to hesitate during the movement. Because it became obvious during the training sessions that most movement times fell between 100 and $150 \mathrm{msec}$, and that hesitation resulted in considerably longer MTs, a criterion of $200 \mathrm{msec}$ was decided upon to eliminate "hesitations" from the analysis of MTs. Thus, trials with MTs longer than $200 \mathrm{msec}$ were categorized as errors. Table 1 shows small differences as a function of stimulus degradation, compatibility, and FPD, but an analysis of variance showed neither significant main effects nor interactions of these variables $(p>.10)$.

\section{DISCUSSION}

These results confirm those of Sternberg (1969) with respect to the additive contributions of stimulus degradation and S-R compatibility to RT. The fact that the differences in experimental setting between Sternberg's and the present study did not change the picture, suggests that the distinction between "encoding" and "response selection" as stages in the choice reaction process is quite robust. Second, the data show that neither stimulus degradation nor S-R compatibility affected the MT. This suggests that, when response execution consists of a short rapid movement, it occurs in succession to the information processing stages contributing to choice RT. It also extends Fitts' (1954) finding that the number of alternatives affects the RT but not the MT.

Regarding the FPD effect, the data show that the effect of FPD on RT was additive to the effects of stimulus degradation and S-R compatibility, and that FPD had no effect on MT. This is consistent with the conclusion that encoding, response selection, and response execution are not affected by FPD.

\section{REFERENCES}

Alegria, J. The time course of preparation after a first peak: Some constraints of reacting mechanisms. Quarterly Journal of Experimental Psychology, 1974, 26, 622-632.

Bertelson, P. The time course of preparation. Quarterly Journal of Experimental Psychology, 1967, 19, 272-279.

FitTs, P. M. The information capacity of the human motor system in controlling the amplitude of movement. Journal of Experimental Psychology, 1954, 47, 381-391.

Posner, M. I., Klein, R., Summers, J., \& Buggie, S. On the selection of signals. Memory \& Cognition, 1973, 1, 2-12.

RAAB, D., Fehrer, E., \& Hershenson, M. Visual reaction time and the Broca-Sulzer phenomenon. Journal of Experimental Psychology, 1961, 61, 193-199.

SANDERS, A. F. Foreperiod duration and the time course of preparation. Acta Psychologica, 1972, 36, 60-71.

SANDERS, A. F. The foreperiod revisited. Quarterly Journal of Experimental Psychology, 1975, 27, 591-598.

SANDERS, A. F. Structural and functional aspects of the reaction process. In S. Dornic (Ed.), Attention and performance VI. Amsterdam: North-Holland, 1977.

STERNBERG, S. On the discovery of processing stages. In W. G. Koster (Ed.), Attention and performance II. Amsterdam: North-Holland, 1969. Reprinted from Acta Psychologica, 1969, 30, 276-315.

TAYLOR, D. A. Stage analysis of reaction time. Psychological Bulletin, 1976, 83, 161-191.

VAN Doorne, H., \& Sanders, A. F. PSARP: A programmable signal and response processor. Behavior Research Methods \& Instrumentation, 1968, 1, 29-32.

(Received for publication April 12, 1978.) 